\title{
A Novel Dual-Band MIMO Antenna with Lower Correlation Coefficient
}

\author{
Jian Zhang, Jun OuYang, Kai Zhi Zhang, and Feng Yang \\ Department of Microwave Engineering, University of Electronic Science and Technology of China (UESTC), Chengdu 610054, China \\ Correspondence should be addressed to Jun OuYang, antenna_ou@163.com
}

Received 18 August 2011; Revised 14 September 2011; Accepted 28 September 2011

Academic Editor: Yuan Yao

Copyright () 2012 Jian Zhang et al. This is an open access article distributed under the Creative Commons Attribution License, which permits unrestricted use, distribution, and reproduction in any medium, provided the original work is properly cited.

This paper demonstrates a novel dual-band operated MIMO antenna which consisted of planar monopole (main antenna) and 3D slot element (auxiliary antenna). The main antenna is printed on a $1.6 \mathrm{~mm}$ thick FR4 board, while the auxiliary antenna is fabricated with gold-coated copper. A lumped impedance network is applied to enhance matching effect at portl. From simulations by commercial software, it can be found that the proposed antenna is able to cover GSM800, GSM900 (lower band), and LTE/ WiMAX/WLAN (higher band) quite well. Good agreements between simulations and measurements are obtained. Corresponding measured results, antenna efficiency, peak gain, and radiation patterns, are presented at the same time. By equipping a passive decoupling element, the coupling power on the ground is radiated into free space, and great enhancement of isolation between antenna elements, especially for lower band, is achieved.

\section{Introdution}

With the prompt development of wireless communication these years, plenty of excellent multimedia services are desirable for users, such as video online or web surfing. So, it is a great challenge worldwide for mobile network operators to accommodate these higher date rates services with current communication systems which possess small channel capacity and poor reliability. Fortunately, it is found that the MIMO (multiple input multiple output) antenna system can be a good candidate to effectively solve this problem. However, it is troublesome that the mutual coupling among closely packed antenna elements will always lead to severe performance deterioration of MIMO systems. In this case, improving the isolation between antenna elements has become a hot topic in the MIMO field, while much effort has been made into studying the technologies for this purpose by antenna experts and scholars.

In order to reduce mutual coupling between MIMO antennas, protruded T-shaped ground plane [1] and corrugated ground plane with $\lambda / 4$ slot [2] are utilized, and modified PIFA with a small local ground plane is proposed in [3]. Chung and Yoon have fabricated a MIMO antenna in which a ground wall and a connecting line are introduced, and it exhibits favorable isolation level [4]. Pairs of slits are etched onto the ground plane of MIMO terminals to avoid current sharing between antenna ports through ground, and significant isolation improvement is obtained compared to conventional ground plane due to the filter effect of etched ground plane [5]. In addition, [6] provides us an different approach for closely packed antennas which consists of a compact integrated diversity antenna with two feed ports. In [7], a MIMO antenna using a T-shaped common grounding element is presented. The proposed MIMO antenna consists of two radiating elements for Mobile-WiMAX service. The structure of T-shaped common grounding element was added between the two modified inverted-L radiating elements to improve the isolation characteristic. To realize the good isolation performance for a small separation, a suspended strip is used to connect the two monopoles together [8], and it forms an effective filter with the ground plane at the required frequency band.

Although there has been many methods to reduce the mutual coupling of MIMO antenna system, just like what we have mentioned above, most of MIMO antennas can only work in a single band, from $1.88 \mathrm{GHz}$ to $2.6 \mathrm{GHz}$ (LTE/ 


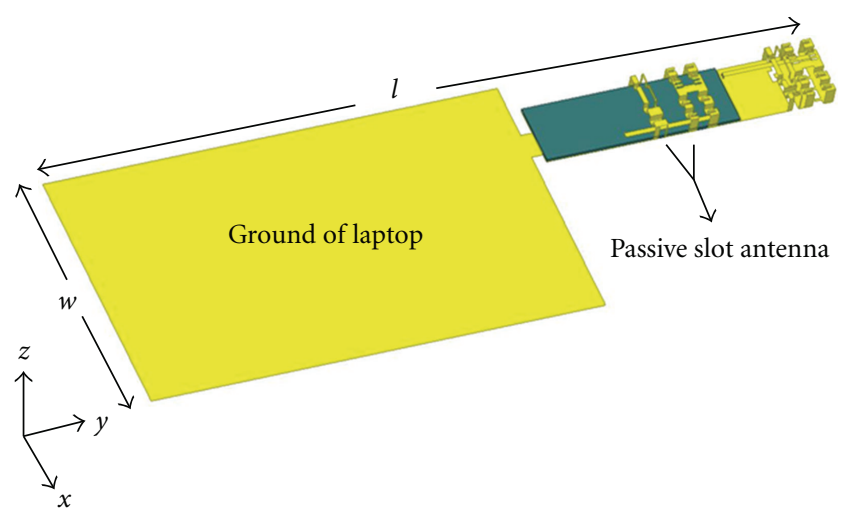

FIgURE 1: Total view of the proposed MIMO antenna with the existence of laptop.

WiMAX/WLAN), and rarely reach to the lower operation bands, such as GSM800 and GSM900, which are still popular bands for our mobile communication at present.

In this paper, a novel USB dongle MIMO antenna with lower correlation coefficient is presented. This MIMO antenna which takes a relative small volume compared with other terminal MIMO antennas consisted of planar monopole and a novel 3D slot element which is meandered in both vertical and horizontal direction [9]. At the same time, for the sake of radiating the coupling power on the ground into free space and thus decreasing the mutual coupling, a passive 3D slot element with separate slots which are responsible for low frequency and high frequency, respectively, is installed in the middle of ground plane. Later, we use the full wave commercial numerical simulation software package to simulate this MIMO antenna and the parameters such as location of the passive antenna, and space between these separate slots have been optimized. It is found that the proposed MIMO antenna works quite well with $S_{11}$ below $-6 \mathrm{~dB}$ both in lower band $(880 \mathrm{MHz}-960 \mathrm{MHz})$ and higher band $(1880 \mathrm{MHz}-2600 \mathrm{MHz})$. Significantly, the isolation for these two antenna ports meets the requirements of handset MIMO system, and especially for lower band, reaches about $-11 \mathrm{~dB}$. At last, the fabricated MIMO antenna is measured in anechoic chamber (SATIMO StarLab), and good agreements have been obtained. Further, to verify the effect of mutual coupling reduction of the decoupling passive slot antenna, some comparisons have been made. It is demonstrated that the proposed terminal MIMO antenna could be an ideal candidate for application in GSM800, GSM900, and LTE/WiMAX/WLAN.

\section{Proposed MIMO Antenna Geometry}

The geometry of the proposed two-channel MIMO antenna is shown in Figure 1. Because ground plays an important role in the performance of the antenna, it has been taken into account for our design.

The details for MIMO antenna are illustrated in Figure 2.

As shown in Figure 2, the grey part is the FR4 board with the thickness $h_{2}$, and the yellow part denotes the copper. The proposed MIMO antenna system comprises planar

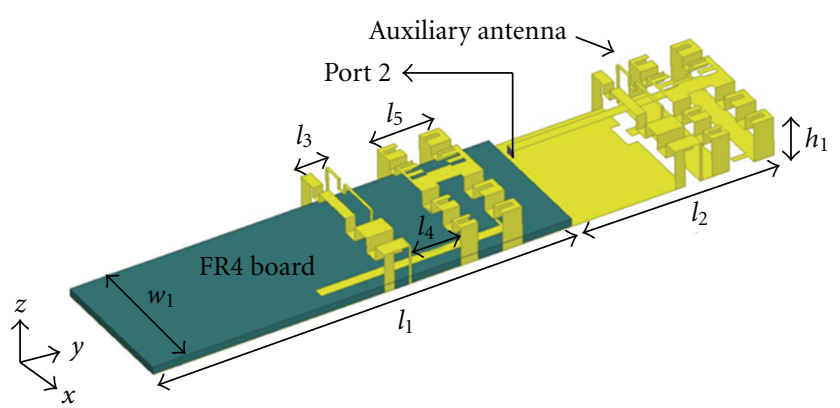

(a)

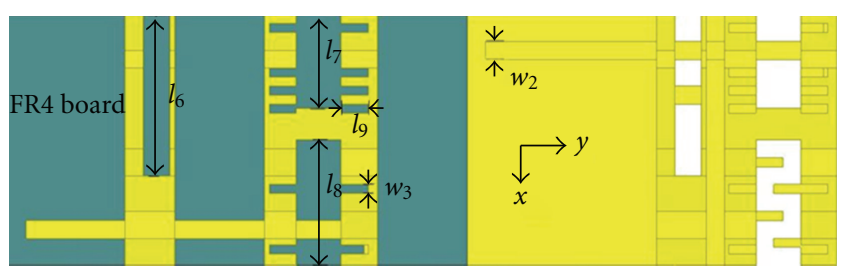

(b)

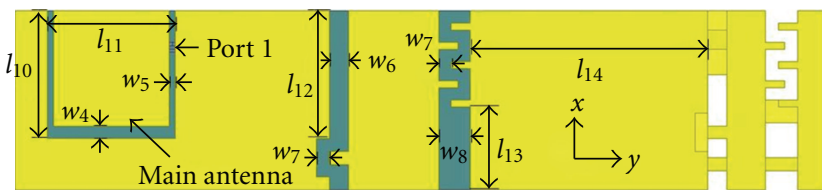

(c)

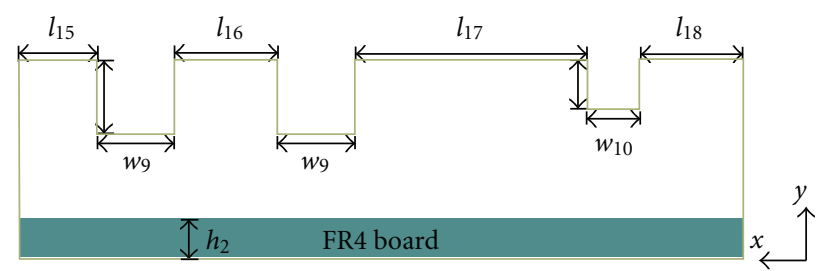

(d)

Figure 2: (a) MIMO antenna structure. (b) Details of the proposed structure (top view). (c) Details of the proposed structure (bottom view). (d) Details of the proposed structure (front side view).

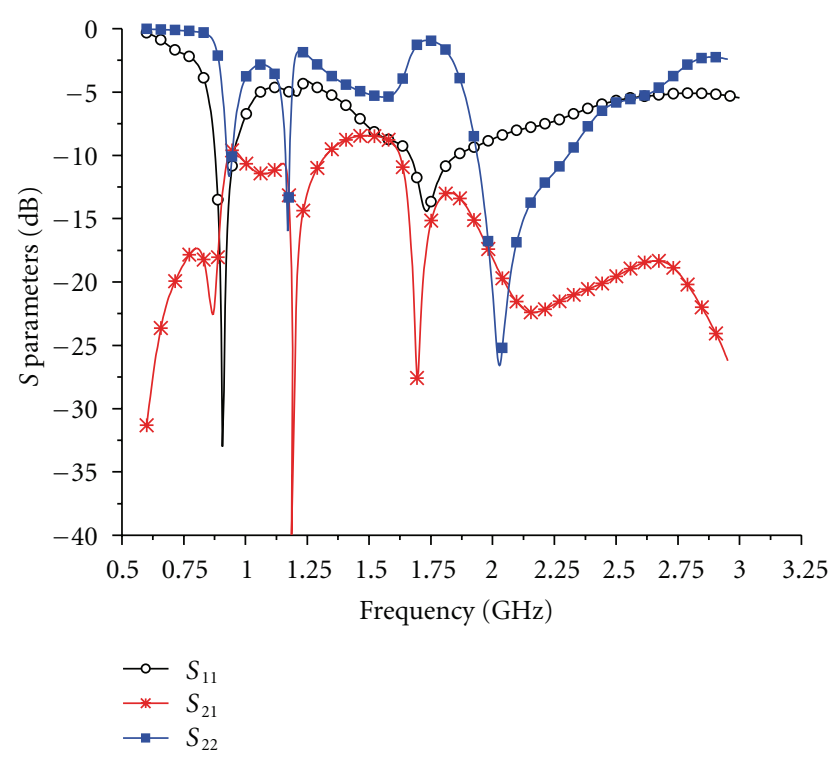

FIGURE 3: Simulated $S$ parameters. 


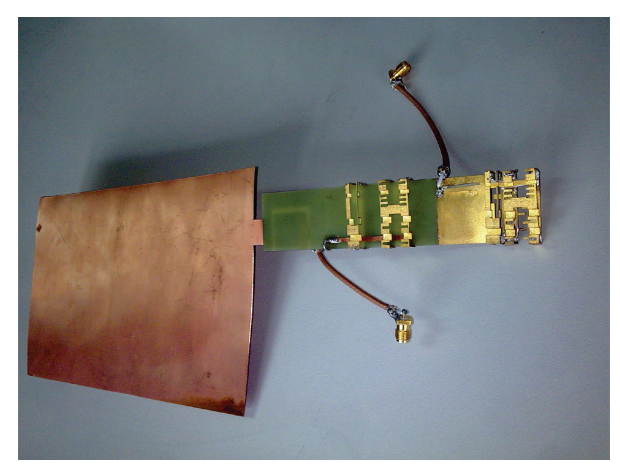

(a) Total view

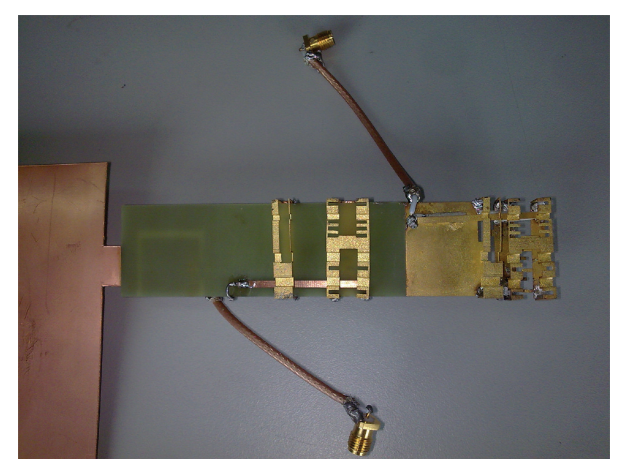

(b) Top view of the MIMO antenna

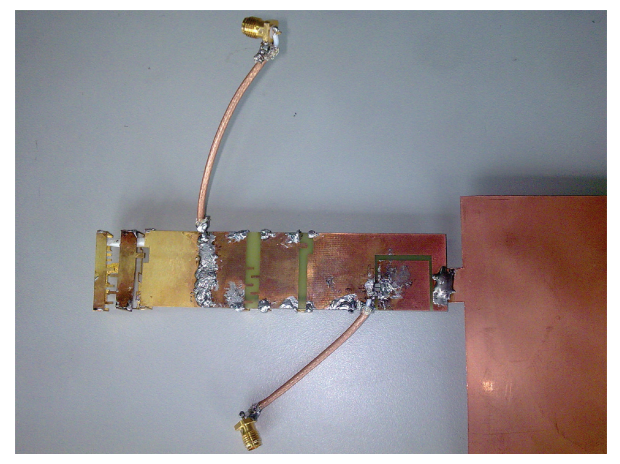

(c) Bottom view of the MIMO antenna

FIGURE 4: Photography of the fabricated MIMO antenna.

TABLE 1: Values of the lumped components.

\begin{tabular}{ll}
\hline Series-capacitance & $4 \mathrm{pF}$ \\
Parallel-capacitance & $7 \mathrm{pF}$ \\
Parallel-inductance & $0.5 \mathrm{nH}$ \\
\hline
\end{tabular}

monopole as main antenna and a novel 3D slot element as auxiliary antenna [9]. The main antenna is printed on the PCB board with the dimensions of $l_{10} \times l_{11}$. The auxiliary antenna located at right side consisted of two horizontally and vertically meandered slots with different resonate lengths; meanwhile, it can be noted that an array of short stubs are placed along these slots to minish the antenna size [10]. A microstrip line with no substrate is used to couple the power to the slots of the auxiliary antenna as feedline. In order to achieve good matching effect, a lumped impedance

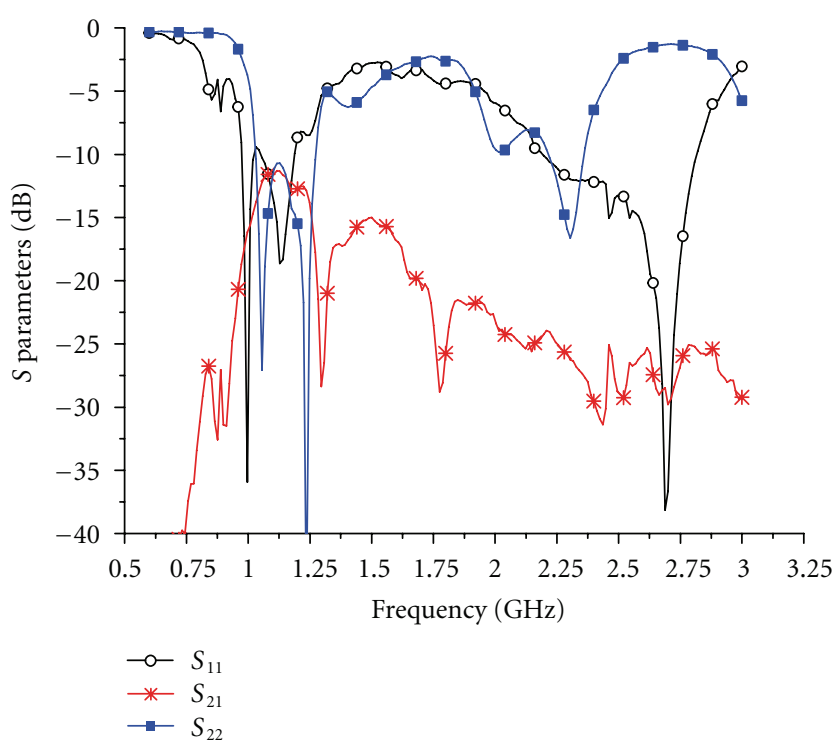

FIGURE 5: Measured $S$ parameters.

network is introduced at Port1 in this design, and the values of the lumped components are given in Table 1.

For multiantenna systems applied in mobile terminals, the mutual coupling between antenna elements is quite severe and mainly caused by ground surface current sharing, especially for low frequency due to relative small space compared to the wavelength. Therefore, to improve the isolation, the preceding $3 \mathrm{D}$ slot element is employed between main antenna and auxiliary antenna to radiate the coupling power on the ground into free space. Finally, for a better performance, the passive 3D slot element is divided into two parts, and the separated slots cover lower band and higher band independently. All the parameters of the antenna structure have been optimized and listed in Table 2.

\section{Simulation and Measured Results}

3.1. Simulation Results. The MIMO antenna model is firstly simulated by the commercial full wave simulation software package-Ansoft HFSS version 13.0, and the simulated $S$ parameters are depicted in Figure 3.

Apparently, from the simulated results, it is found that both main antenna and auxiliary antenna can work well for dual-band operation with reflection $\left(S_{11}\right.$ and $\left.S_{22}\right)$ lower than $-6 \mathrm{~dB}$, which is acceptable for the applications of miniaturized terminal. Moreover, the MIMO antenna system possess a good isolation level about $-10 \mathrm{~dB}$ for lower band $(880 \mathrm{MHz}-960 \mathrm{MHz})$ and average $-20 \mathrm{~dB}$ for higher band (1880 MHz-2600 MHz).

3.2. Measured Results. The fabricated antenna is shown in Figure 4, and the copper thickness is $0.5 \mathrm{~mm}$.

We measured this antenna in the anechoic chamber (SATIMO StarLab). The measured data, including efficiency, peak gain, and radiation patterns, are obtained by feeding one port while the other one terminated with a $50 \mathrm{Ohms}$ load. 


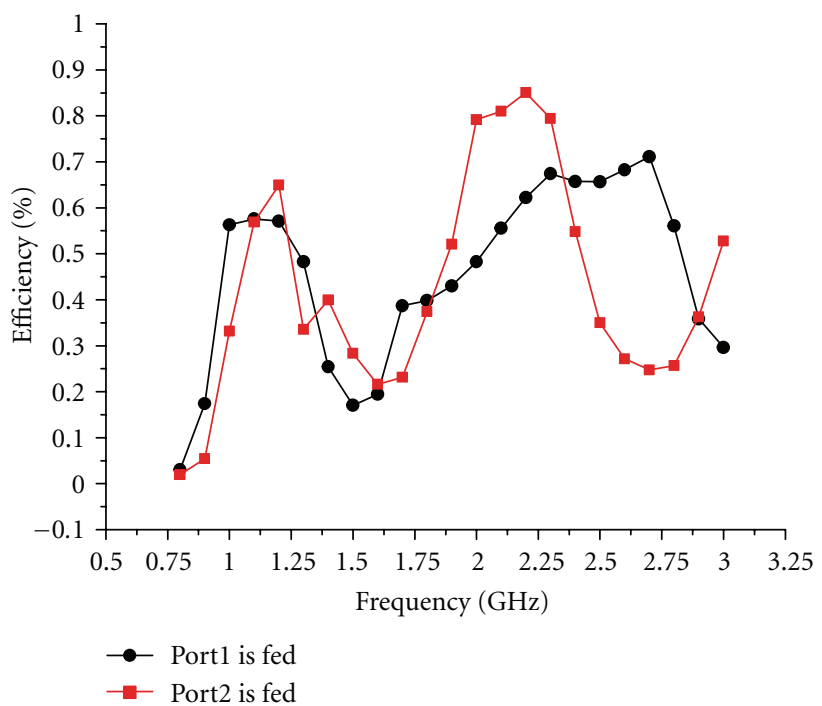

(a) Measured efficiency

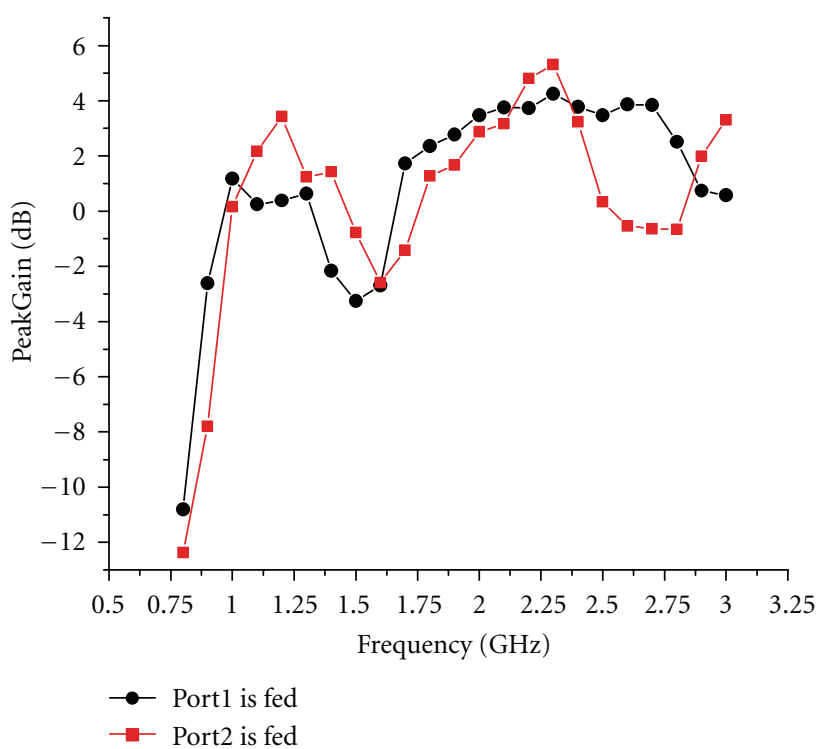

(b) Measured peak gain

FIgURE 6: Measured efficiency and peak gain.

TABLE 2: Parameters of the antenna structure.

\begin{tabular}{lccccccccc}
\hline$l$ & $331 \mathrm{~mm}$ & $l_{5}$ & $12.5 \mathrm{~mm}$ & $l_{10}$ & $20 \mathrm{~mm}$ & $l_{15}$ & $3 \mathrm{~mm}$ & $w_{1}$ & $28 \mathrm{~mm}$ \\
$l_{1}$ & $85 \mathrm{~mm}$ & $l_{6}$ & $21.5 \mathrm{~mm}$ & $l_{11}$ & $20 \mathrm{~mm}$ & $l_{16}$ & $4 \mathrm{~mm}$ & $w_{2}$ & $2 \mathrm{~mm}$ \\
$l_{2}$ & $41 \mathrm{~mm}$ & $l_{7}$ & $10.5 \mathrm{~mm}$ & $l_{12}$ & $20 \mathrm{~mm}$ & $l_{17}$ & $9 \mathrm{~mm}$ & $w_{3}$ & $1 \mathrm{~mm}$ \\
$l_{3}$ & $5.5 \mathrm{~mm}$ & $l_{8}$ & $14 \mathrm{~mm}$ & $l_{13}$ & $13 \mathrm{~mm}$ & $l_{18}$ & $4 \mathrm{~mm}$ & $w_{4}$ & $2 \mathrm{~mm}$ \\
$l_{4}$ & $10 \mathrm{~mm}$ & $l_{9}$ & $3 \mathrm{~mm}$ & $l_{14}$ & $37 \mathrm{~mm}$ & $w$ & $120 \mathrm{~mm}$ & $w_{5}$ & $1 \mathrm{~mm}$ \\
$w_{6}$ & $3 \mathrm{~mm}$ & $w_{7}$ & $2 \mathrm{~mm}$ & $w_{8}$ & $5 \mathrm{~mm}$ & $w_{9}$ & $3 \mathrm{~mm}$ & $w_{10}$ & $2 \mathrm{~mm}$ \\
$h_{1}$ & $8 \mathrm{~mm}$ & $h_{2}$ & $1.6 \mathrm{~mm}$ & & & & & & \\
\hline
\end{tabular}

Comparing Figures 3 and 5, even though the resonate point of auxiliary antenna shifts a little higher due to the error which comes from fabrication and installation, it cannot be denied that there is good agreement between simulation and measurement $\left(S_{11}, S_{22}<-6 \mathrm{~dB}, S_{21}<-11 \mathrm{~dB}\right)$.

Figure 6 demonstrates that the proposed MIMO antenna operates quite efficiently, at least $40 \%$ for lower band and peak efficiency appearing within higher band, is approximately $88 \%$. The peak gains of the antenna are also qualified to the desired applications. Radiation patterns for lower and higher band are drawn in Figures 7 and 8.

3.3. Correlation Coefficients. In order to further investigate whether these two channels are independent enough, we use the following formula to compute the correlation coefficients of the proposed MIMO antenna:

$$
\rho_{e}=\frac{\left(\oint \oint\left(X P R \cdot E_{1 \theta}(\theta, \phi) \cdot E_{2 \theta}^{*}(\theta, \phi) \cdot \mathcal{A}+E_{1 \phi} \bullet(\theta, \phi) \bullet E_{2 \phi}^{*}(\theta, \phi) \cdot \mathscr{B}\right) \cdot \sin \theta \cdot d \theta d \phi\right)^{2}}{\oint \oint\left(X P R \cdot G_{1 \theta}(\theta, \phi) \cdot \mathcal{A}+G_{1 \phi}(\theta, \phi) \cdot \mathcal{B}\right) \cdot \sin \theta \cdot d \theta d \phi \cdot \oint \oint\left(X P R \cdot G_{2 \theta}(\theta, \phi) \cdot \mathcal{A}+G_{2 \phi} \cdot(\theta, \phi) \cdot \mathcal{B}\right) \cdot \sin \theta \cdot d \theta d \phi},
$$

where $\mathcal{A}$ denotes $P_{\theta}(\theta, \phi)$ and $\mathscr{B}$ denotes $P_{\phi}(\theta, \phi)$.

In the formula above, $G_{\theta}=E_{\theta}(\theta, \phi) \bullet E_{\theta}^{*}(\theta, \phi), G_{\phi}=$ $E_{\phi}(\theta, \phi) \bullet E_{\phi}^{*}(\theta, \phi) ; E_{1 \theta}(\theta, \phi)$ and $E_{2 \theta}(\theta, \phi)$ are plural radiation patterns of main antenna and auxiliary antenna, respectively, for vertical polarization situation; $E_{1 \phi}(\theta, \phi)$ and $E_{2 \phi}(\theta, \phi)$ are for horizontal polarization case; $P_{\theta}(\theta, \phi)$ and $P_{\phi}(\theta, \phi)$ denote power probability distributions of two incident wave with different polarizations, also the distributions vary with various communication networks and operation circumstance of antennas. On the other hand,

$$
\begin{aligned}
& P_{\theta}(\theta, \phi)=P_{\theta}(\theta) \cdot P_{\theta}(\phi), \\
& P_{\phi}(\theta, \phi)=P_{\phi}(\theta) \cdot P_{\phi}(\phi),
\end{aligned}
$$

$P_{\theta}(\theta)$ and $P_{\phi}(\theta)$ refer to power probability distributions of incident wave in vertical direction, and $P_{\theta}(\phi)$ and $P_{\phi}(\phi)$ 


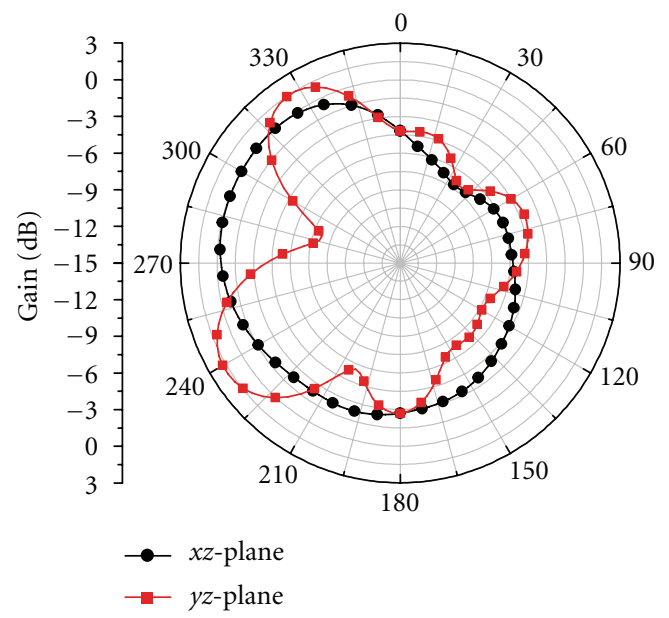

(a) $1100 \mathrm{MHz}$

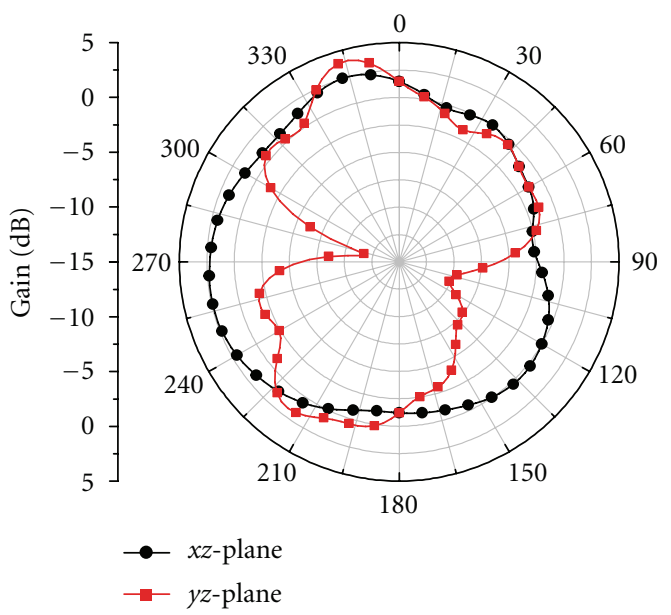

(b) $2200 \mathrm{MHz}$

Figure 7: Port1 stimulated.

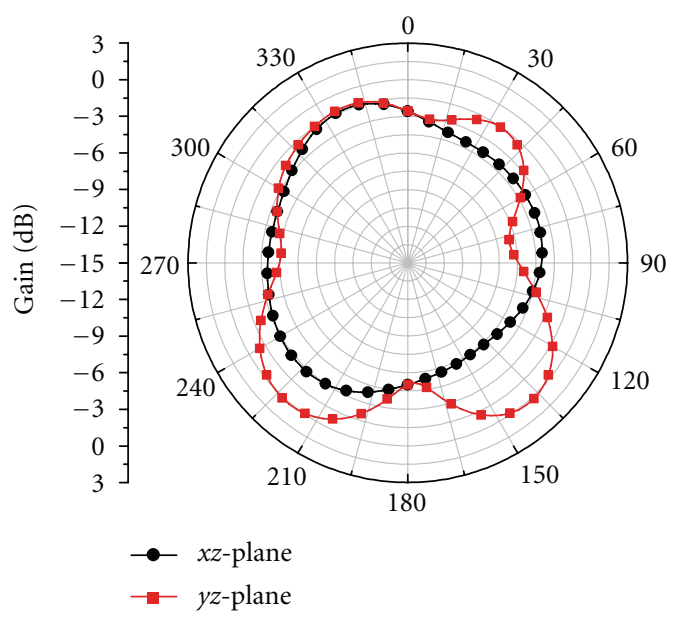

(a) $1100 \mathrm{MHz}$

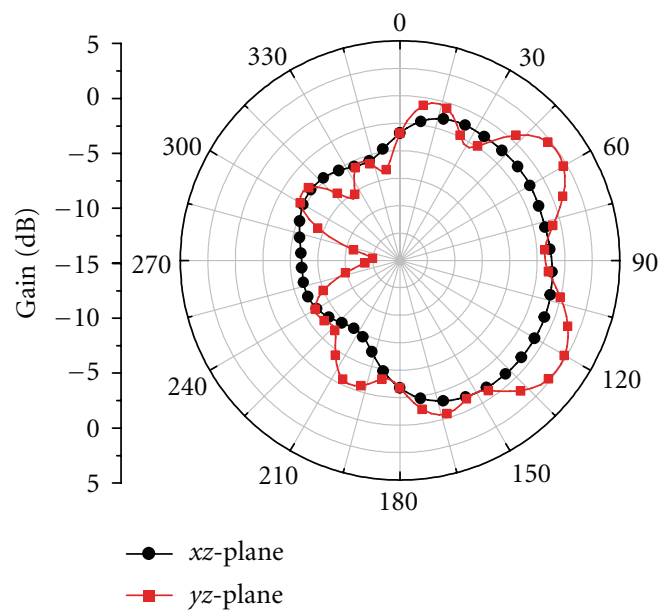

(b) $2200 \mathrm{MHz}$

FIgURE 8: Port2 stimulated.

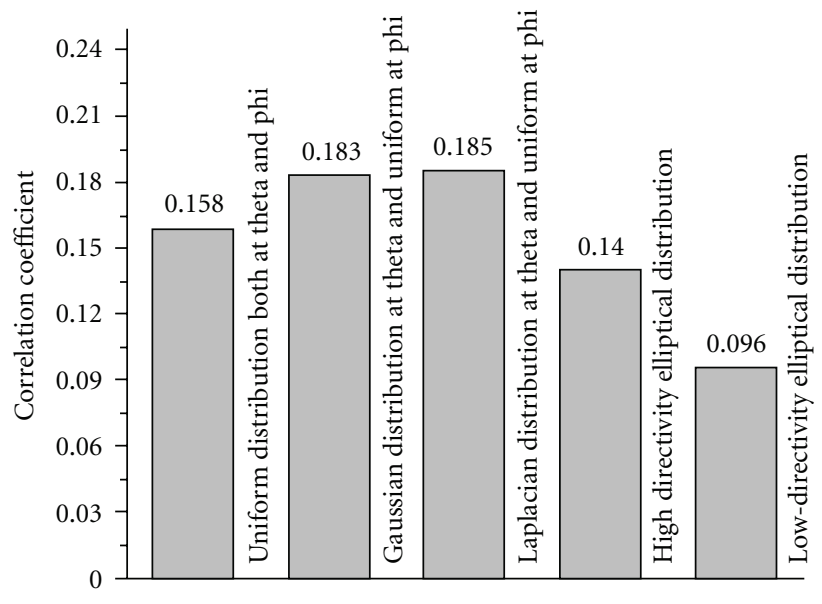

(a)

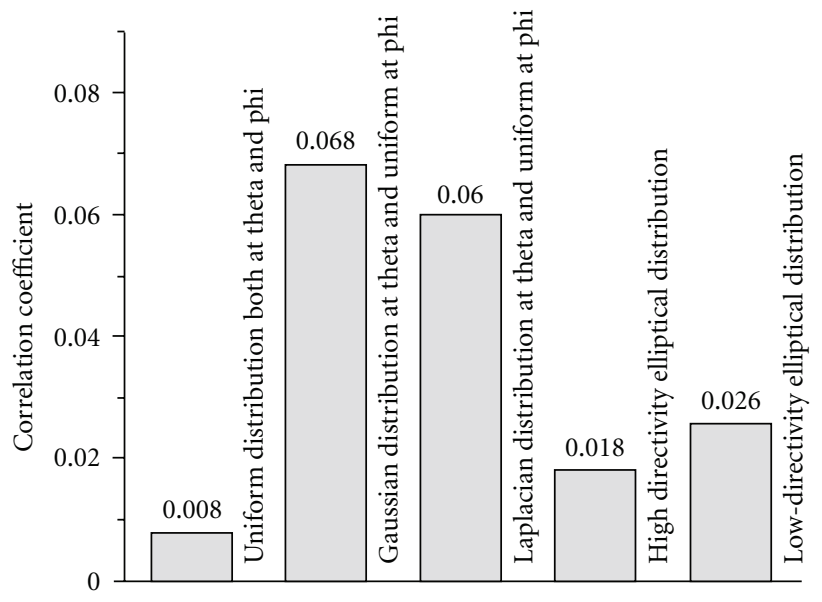

(b)

Figure 9: Correlation coefficient: (a) (1100 MHz); (b) (2200 MHz). 


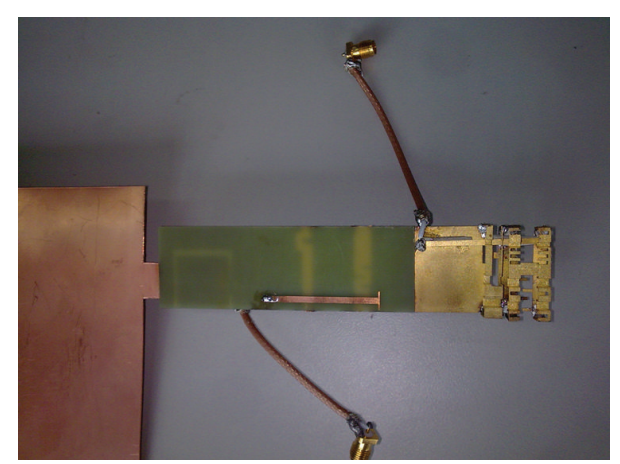

(a) Top view

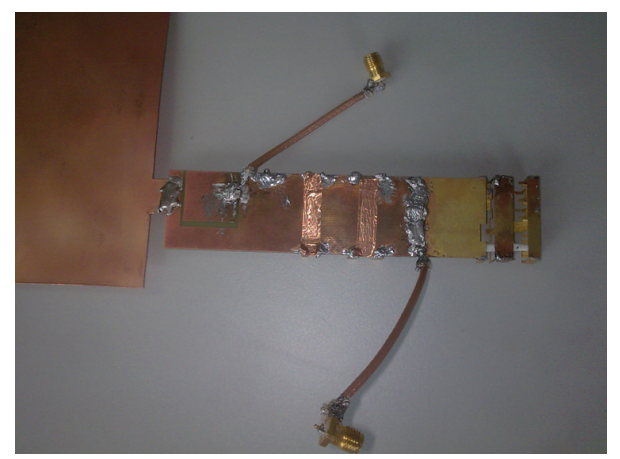

(b) Back view

FIgURE 10: Transformed MIMO antenna.

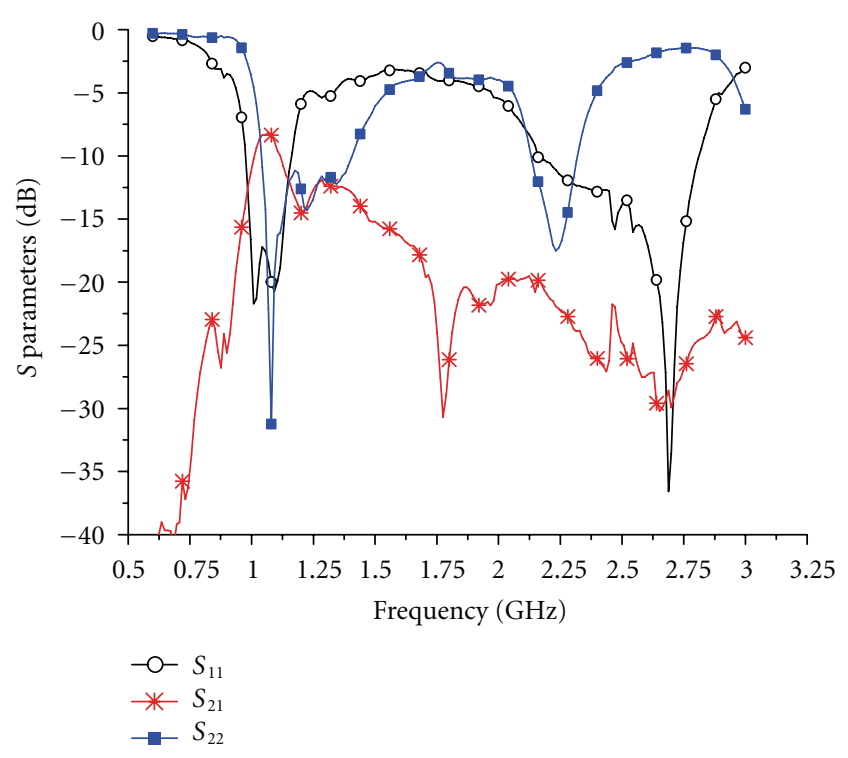

FIGURE 11: Measured $S$ parameters of transformed MIMO antenna.

refer to power probability distributions of incident wave in horizontal direction.

The calculated correlation coefficients of these two antenna elements are diagramed in Figure 9.

Five different correlation coefficients are calculated with five distinct distributions, which are uniform distribution both at theta and phi, Gaussian distribution at theta and uniform at phi, Laplacian distribution at theta and uniform at phi, high-directivity elliptical distribution, and low-directivity elliptical distribution, respectively. The corresponding equations of those probability distributions are provided in [11].

No matter which kind of distributions is chosen, the calculated correlation coefficients are extremely low here, particularly for higher operation band, all of which are below 0.07 , and even the maximal one is merely 0.068 . Therefore, these two antenna elements can be reasonably regarded as independent channels to provide better channel capacity.

3.4. Verifying the Effect of the Passive Decoupling Element. To verify the effect of the passive decoupling element, the passive decoupling element is removed away from the proposed MIMO antenna. The transformed antenna is shown in Figure 10.

As illustrated in Figure 10, the decoupling passive 3D slot element has been removed away and the original slot etched on the antenna ground is covered by copper foil. The measured $S$ parameters for this case are demonstrated in Figure 11.

It can be easily found that a deterioration of isolation of the antenna elements occurs comparing with Figure 5, especially for lower band of which the worst point climbs to about $-7.5 \mathrm{~dB}$ from $-11 \mathrm{~dB}$. The correlation coefficient of the transformed MIMO antenna has also increased to a high level, from the original 0.096 to 0.2535 at $1100 \mathrm{MHz}$ with the low-directivity elliptical probability distribution. Thus, we can get to the conclusion that the passive decoupling element is capable of greatly reducing the mutual couple for MIMO antenna.

\section{Conclusions}

A novel dual-band operated MIMO antenna consisted by planar monopole and 3D slot element is presented in this paper. As a lumped impedance network is introduced in port1, both main antenna and auxiliary antenna matches very well no matter lower band or higher band. Since the coupling power on the ground is radiated into free space by a passive decoupling element, great enhancement of the isolation between antenna elements is obtained. This novel MIMO antenna is a good candidate for the mobile terminal application on GSM800, GSM900, and LTE/WiMAX/WLAN.

\section{Acknowledgments}

This work was supported by the postdoctoral Science Foundation of China (no. 20090461325 and no. 201003690), the Natural Science Foundation of China (no. 61001029 and no. 10876007), and the Fundamental Research Funds for the Central Universities (no. 103.1.2 E022050205).

\section{References}

[1] T. Y. Wu, S. T. Fang, and K. L. Wong, "Printed diversity monopole antenna for WLAN operation," Electronics Letters, vol. 38, no. 25, pp. 1625-1626, 2002. 
[2] M. Karakoikis, C. Soras, G. Tsachtsiris, and V. Makios, "Compact dual-printed inverted-F antenna diversity systems for portable wireless devices," IEEE Antennas and Wireless Propagation Letters, vol. 3, no. 1, pp. 9-13, 2004.

[3] Y. Gao, C. C. Chiau, X. Chen, and C. G. Parini, "Modified PIFA and its array for MIMO terminals," pp. 255-259.

[4] K. Chung and J. H. Yoon, "Integrated MIMO antenna with high isolation characteristic," Electronics Letters, vol. 43, no. 4, pp. 199-201, 2007.

[5] C. Y. Chiu, C. H. Cheng, R. D. Murch, and C. R. Rowell, "Reduction of mutual coupling between closely-packed antenna elements," IEEE Transactions on Antennas and Propagation, vol. 55, no. 6, pp. 1732-1738, 2007.

[6] S. C. K. Ko and R. D. Murch, "Compact integrated diversity antenna for wireless communications," IEEE Transactions on Antennas and Propagation, vol. 49, no. 6, pp. 954-960, 2001.

[7] J. Kwon, D. Kim, Y. Lee, and J. Choi, "Design of a MIMO antenna for USB dongle application using common grounding," in Proceedings of the 13th International Conference on Advanced Communication Technology (ICACT '11), pp. 313316, Seoul, South Korea, 2011.

[8] Z. Li, M. -S. Han, X. Zhao, and J. Choi, "MIMO antenna with isolation enhancement for wireless USB dongle application at WLAN band," in Proceedings of the Asia-Pacific Microwave Conference Proceedings (APMC '10), pp. 758-761, Yokohama, Japan, December 2010.

[9] J. OuYang, J. Zhang, K. Z. Zhang, and F. Yang, "Compact folded dual-band slot antenna for wireless communication USB dongle application," Journal of Electromagnetic Waves and Applications, vol. 25, no. 8-9, pp. 1221-1230, 2011.

[10] N. Behdad and K. Sarabandi, "Bandwidth enhancement and further size reduction of a class of miniaturized slot antennas," IEEE Transactions on Antennas and Propagation, vol. 52, no. 8, pp. 1928-1935, 2004.

[11] V. Plicanic, Antenna diversity studies and evaluation, M.S. thesis, Master of Science Thesis Vanja Plicanic in Cooperation with Ericsson Mobile Communications AB, 2004. 

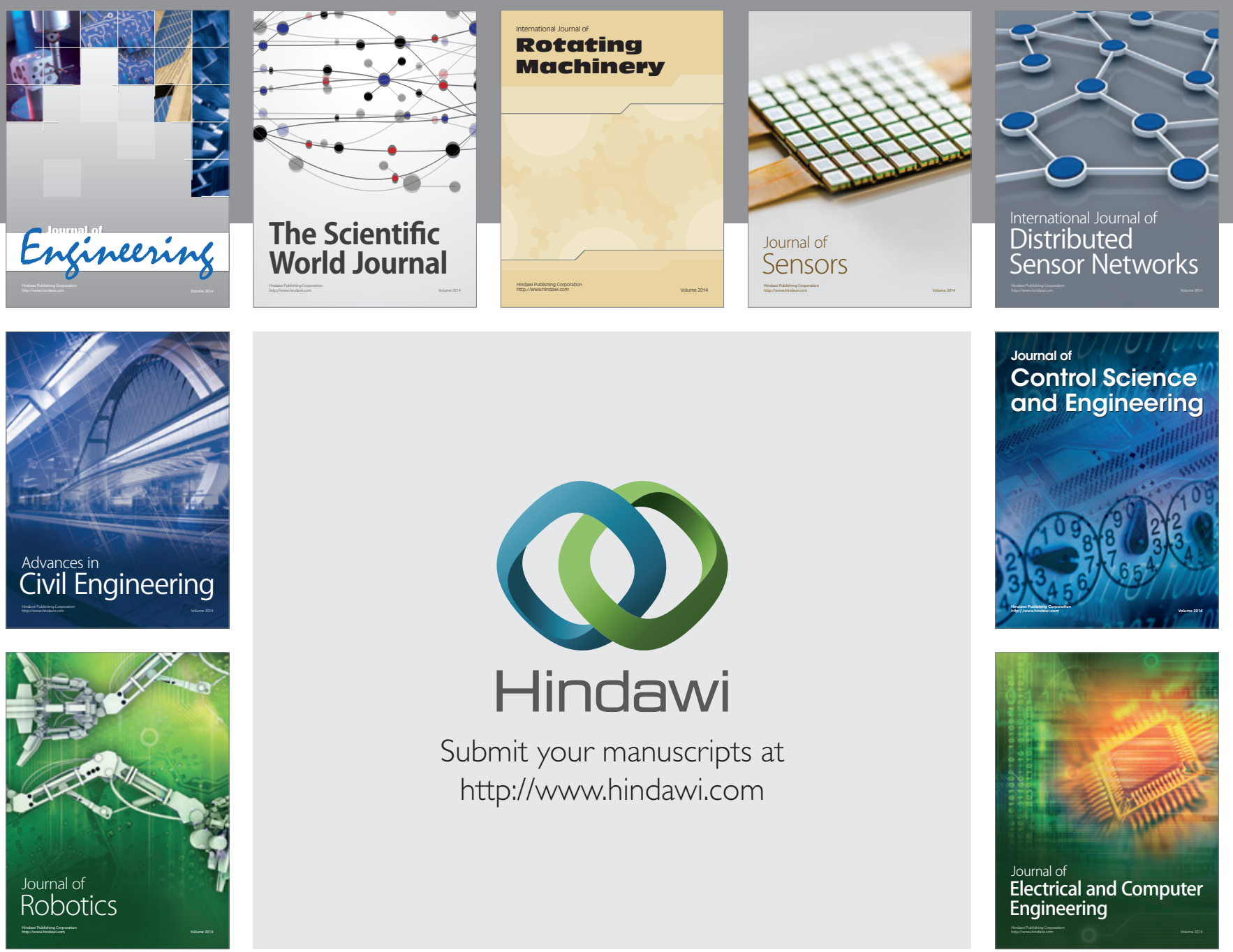

Submit your manuscripts at

http://www.hindawi.com
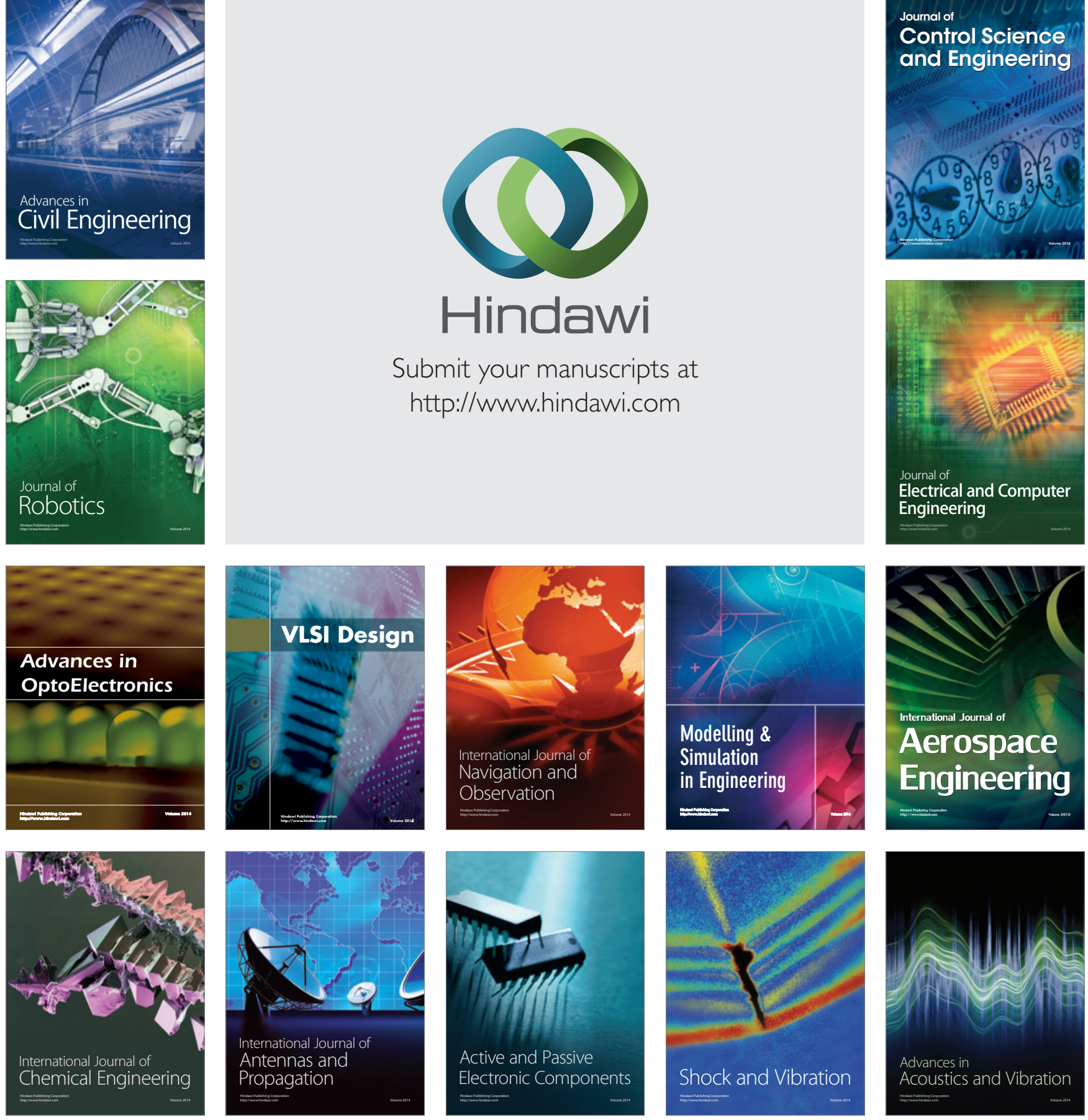